\title{
Short Note on "Nonlinear Optimization Problem Subjected to Fuzzy Relational Equations Defined by Dubois-Prade Family of t-Norms"
}

\author{
Xiaoling LIU ${ }^{\mathrm{a}}$, Khizar HAYAT ${ }^{\mathrm{b}}$ and Xiaopeng YANG ${ }^{\mathrm{a}, 1}$ \\ ${ }^{a}$ School of Mathematics and Statistics, Hanshan Normal University, Chaozhou, China \\ ${ }^{\mathrm{b}}$ Department of Mathematics, University of Kotli, Azad Jammu and Kashmir, Pakistan
}

\begin{abstract}
This short note aims to make some modification for improving the results presented in [1]. A. Ghodousian et al. discussed the resolution of a system of maxDubois-Prade fuzzy relation equations based on their proposed index sets $J_{i}, i \in I$. It is found that not every $e \in E=J_{1} \times J_{2} \times \cdots \times J_{m}$ corresponds to a solution. To overcome this flaw, we modify the expression of the index sets, denoted by $J_{i}^{=}$, $i \in I$. Based on the modified index sets, resolution of the max-Dubois-Prade fuzzy relation equations becomes easier, regarding the computational cost.
\end{abstract}

Keywords. fuzzy relation equation, max-Dubois-Prade composition, solution set, minimal solution

\section{Introduction}

In a recent paper [1], A. Ghodousian et al. studied the nonlinear optimization problem subject to a system of max-Dubois-Prade fuzzy relation equations as follows

$$
\begin{aligned}
& \min f(x) \\
& \text { s.t. } A \varphi x=b, \\
& \quad x \in[0,1]^{n} .
\end{aligned}
$$

Denote the index sets $I=\{1,2, \cdots, m\}$ and $J=\{1,2, \cdots, n\}$. In above the fuzzy relation equations system

$$
A \varphi x=b,
$$

$A=\left(a_{i j}\right)_{m \times n} \in[0,1]^{m \times n}$ represents a fuzzy matrix, while $b=\left(b_{i}\right)_{m \times 1} \in[0,1]^{m}$ is a $m$ dimensional fuzzy vector. The max-Dubois-Prade composition $\varphi$ is defined as

\footnotetext{
${ }^{1}$ Corresponding Author: Xiaopeng Yang; E-mail: 706697032@qq.com. This work was supported by the National Natural Science Foundation of China (61877014) and the funds provided by the Education Department of Guangdong Province (2017KTSCX124, 2019KZDXM013, ZD201802).
} 


$$
\varphi(x, y)=T_{D P}^{\gamma}(x, y)=\frac{x y}{\max \{x, y, \gamma\}}, \forall x, y \in[0,1],
$$

where $\gamma \in(0,1)$ is some fixed parameter. Notice that $T_{D P}^{0}(x, y)=\min \{x, y\}$ and $T_{D P}^{1}(x, y)=x y$. That is to say, when $\gamma=0$, the composition $\varphi=T_{D P}^{0}$ becomes "min", while $\gamma=0, \varphi=T_{D P}^{1}$ turns out to be "product" composition. The authors first presented the resolution of the constraint, i.e. system (2). Based on the properties of the solution set of (2), they further applied the genetic algorithm to search an approximate optimal solution of problem (1). After carefully examination, we found that some of the result in [1] could be improved, for making it always true for the general case.

Searching all the solutions of a fuzzy relation system, including equations system or inequalities system, is an lasting research topic [2]-[6]. In the resolution of a fuzzy relation system, the composition plays important role. The composition has been extended from the initial max-min operator to the general max-t-norm one. Recently, fuzzy relation systems with addition-min [4]-[6] or max-Dubois-Prade [1,7] were also investigated. Especially, the solution set of consistent fuzzy relation inequalities system with additionmin composition is no longer non-convex, but convex [4]. Moreover, the number of its minimal solutions (when non-unique) are no longer finite, but infinite [4]. However, resolution of all its solutions remains an open problem [5].

When applying the fuzzy relation system in some application field, the relevant optimization problem might be dealt. S.-C. Fang and G. Li first introduced the optimization problem minimizing a linear function with fuzzy relation equations constraint [8]. There are much research focusing on such a topic [8]-[11]. The Branch-and-Bounded approach is one of the most applied resolution methods. However, it is no longer suit for optimization problem with addition-min composition $[10,11,12]$. For fuzzy relation programming with general nonlinear objective function, the generic algorithm is an common method to search its approximate optimal solution $[1,7,13,14]$. Computation complexity and accuracy are important criterions for evaluation of a generic algorithm. Other specific fuzzy relation nonlinear programming problems could be handled according to their special objective functions and structure of the feasible regions [15]-[20].

The optimization problem with a linear objective function and a group of maxproduct fuzzy relation equation constraints was first studied by J. Loetamonphong and S.-C. Fang [21]. The imitated the resolution approach presented in [8]. In recent years, the fuzzy relation inequalities system with max-product composition was further applied to the foodstuff management in a given city [20,22,23] and the wireless communication station system $[24,25,26]$. The linear objective function was replaced by a nonlinear one [22,23,24,25]. Obviously, the resolution method presented in [21] was no longer effective for those nonlinear fuzzy relation optimization problems [22,23,24,25]. J. Qiu et al. further investigated the fuzzy relation optimization problem with a bi-level objective function [26]. Fuzzy relation inequality with addition-min composition was another challenging researching topic. As pointed out in [4], in most cases, a system of additionmin fuzzy relation inequalities has an infinite number of minimal solutions. As we know, there is no efficient method for obtaining all the minimal solutions till now. Hence, one is not able to find its complete solution set. However, searching some specific solution$\mathrm{s}$ of the addition-min fuzzy relation inequalities is interesting and achievable. Several resolution method were proposed for the optimization problem with a linear objective function and the addition-min fuzzy relation inequality constraints $[10,11,27]$. For decreasing the network congestion in the P2P file sharing system, X. Yang et al. [12,28,29] 
further established and investigated the corresponding min-max programming subjec$\mathrm{t}$ to the addition-min fuzzy relation inequalities system. Moreover, the relevant multiple objective optimization problem was also studied, which allowed the system manager to consider three objectives (system congestion, cost, and penalty) simultaneously [30]. Besides, some other issues with respect to the addition-min fuzzy relation inequalities system were introduced and investigated [31,32].

The contribution and novelty of this work are summarized as follows.

(i) Point out the drawbacks of the existing work [1].

The following Theorem 2 and its relevant assertion are adopted from [1] (see pages $169-170$ in [1]).

- Theorem 2. $S_{T_{D P}^{\gamma}}(A, b)=\bigcup_{e \in E}[\underline{X}(e), \bar{X}]$.

- As a consequence, it turns out that $\bar{X}$ is the unique maximum solution and $\underline{X}(e)$, $(e \in E)$ are the minimal solutions of $S_{T_{D P}^{\gamma}}(A, b)$. Moreover, we have the following corollary that is directly resulted from Theorem 2.

We have found that the results in the above "Theorem 2" and its relevant assertion might be incorrect.

(ii) Give the reason causing the above drawbacks.

In fact, $\underline{X}(e)$ might not be a feasible solution of problem (1). In other words, it is possible that $\underline{X}(e) \notin S_{T_{D P}^{\gamma}}^{\gamma}(A, b)$. This is the reason causing the above-presented drawbacks. If $\underline{X}(e) \notin S_{T_{D P}^{\gamma}}(A, b)$ holds, then $\underline{X}(e)$ is not a solution of system (2). As a consequence, the result that $S_{T_{D P}^{\gamma}}(A, b)=\bigcup_{e \in E}[\underline{X}(e), \bar{X}]$, in Theorem 2, is invalid. In addition, $\underline{X}(e)$ is not a minimal solution of $S_{T_{D P}^{\gamma}}(A, b)$.

(iii) Make some corresponding corrections.

In this work, we have made some modifications to the index sets $J_{1}, J_{2}, \cdots, J_{m}$ and the Definition 4 in [1]. The modified index sets are denoted by $J_{1}^{=}, J_{2}^{=}, \cdots, J_{m}^{=}$. After these modifications, it has been further proved that for any $e \in E^{=}=J_{1}^{=} \times J_{2}^{=} \times \cdots \times J_{m}^{=}$, it holds that $\underline{X}(e) \in S_{T_{D P}^{\gamma}}(A, b)$. Consequently, the corresponding results in the above theorem and assertion turn out to be correct.

We organized our paper as follows. Section 2 provides some necessary preliminaries on the max-Dubois-Prade fuzzy relation equations. The main results of this paper are set in Section 2, including three parts: Phenomenon, Reason and Correction. Section 4 is the simple conclusion.

\section{Preliminaries}

In this section we recall some concepts and results which are presented in [1].

The max-Dubois-Prade fuzzy relation equations (2) could be written as

$$
\varphi\left(a_{i}, b_{i}\right)=\varphi\left(a_{i 1}, x_{1}\right) \vee \varphi\left(a_{i 2}, x_{2}\right) \vee \cdots \vee \varphi\left(a_{i n}, x_{n}\right)=b_{i}, \quad i \in I,
$$

where $\varphi\left(a_{i j}, x_{j}\right)=\frac{a_{i j} x_{j}}{\max \left\{a_{i j}, x_{j}, \gamma\right\}}$.

Let

$$
S_{T_{D P}^{\gamma}}^{\gamma}(A, b)=\left\{x \in[0,1]^{n} \mid A \varphi x=b\right\}
$$


be the complete solution set of system (2). Denote the solution set of the $i$ th equation of system (2), i.e.

$$
\varphi\left(a_{i 1}, x_{1}\right) \vee \varphi\left(a_{i 2}, x_{2}\right) \vee \cdots \vee \varphi\left(a_{i n}, x_{n}\right)=b_{i}
$$

by $S_{T_{D P}^{\gamma}}\left(a_{i}, b_{i}\right)$, for all $i \in I$. Then it is clear that

$$
S_{T_{D P}^{\gamma}}^{\gamma}(A, b)=\bigcap_{i \in I} S_{T_{D P}^{\gamma}}\left(a_{i}, b_{i}\right)
$$

Definition 1. For each $i \in I$, we define the following index sets

$$
\begin{aligned}
& J_{i}^{1,1}=\left\{j \in J: a_{i j}>b_{i} \text { and } \gamma>a_{i j}\right\}, \\
& J_{i}^{1,2}=\left\{j \in J: a_{i j}>b_{i} \text { and } \gamma \leq a_{i j}\right\}, \\
& J_{i}^{2,1}=\left\{j \in J: a_{i j}=b_{i} \text { and } b_{i} \neq 0\right\}, \\
& J_{i}^{2,2}=\left\{j \in J: a_{i j}=b_{i}=0\right\} .
\end{aligned}
$$

Let $J_{i}=J_{i}^{1,1} \cup J_{i}^{1,2} \cup J_{i}^{2,1} \cup J_{i}^{2,2}$. Additionally we set

$$
l_{i j}=\left\{\begin{array}{ll}
\frac{\gamma b_{i}}{a_{i j}} & j \in J_{i}^{1,1}, \\
b_{i} & j \in J_{i}^{1,2}, \\
\max \left\{b_{i}, \gamma\right\} & j \in J_{i}^{2,1}, \\
0 & j \in J_{i}^{2,2},
\end{array} \quad \text { and } \quad u_{i j}= \begin{cases}\frac{\gamma b_{i}}{a_{i j}} & j \in J_{i}^{1,1}, \\
b_{i} & j \in J_{i}^{1,2}, \\
1 & j \in J_{i}^{2,1} \cup J_{i}^{2,2}\end{cases}\right.
$$

Lemma 1. Let $i \in$ I. If $j \notin J_{i}$, then $T_{D P}^{\gamma}\left(a_{i j}, x_{j}\right)<b_{i}, \forall x_{j} \in[0,1]$.

Lemma 2. Suppose that $i \in I$ and $j \in J_{i}$.

(a) $x_{j}>u_{i j}$ iff $T_{D P}^{\gamma}\left(a_{i j}, x_{j}\right)>b_{i}$.

(b) $x_{j}<l_{i j}$ iff $T_{D P}^{\gamma}\left(a_{i j}, x_{j}\right)<b_{i}$.

(c) $l_{i j} \leq x_{j} \leq u_{i j}$ iff $T_{D P}^{\gamma}\left(a_{i j}, x_{j}\right)=b_{i}$.

Lemma 3. For a fixed $i \in I, S_{T_{D P}^{\gamma}}\left(a_{i}, b_{i}\right) \neq \emptyset$ if and only if $J_{i} \neq \emptyset$.

Definition 2. Suppose that $i \in I$ and $S_{T_{D P}^{\gamma}}\left(a_{i}, b_{i}\right) \neq \emptyset$ (hence, $J_{i} \neq \emptyset$ from Lemma 3). Let $\hat{x}_{i}=\left[\left(\hat{x}_{i}\right)_{1},\left(\hat{x}_{i}\right)_{2}, \cdots,\left(\hat{x}_{i}\right)_{n}\right] \in[0,1]^{n}$ where the components are defined as follows:

$$
\left(\hat{x}_{i}\right)_{k}=\left\{\begin{array}{ll}
u_{i j} & k \in J_{i}, \\
1 & k \notin J_{i},
\end{array} \quad \forall k \in J .\right.
$$

Also, for each $j \in J_{i}$, we define $\breve{x}_{i}(j)=\left[\breve{x}_{i}(j)_{1}, \breve{x}_{i}(j)_{2}, \cdots, \breve{x}_{i}(j)_{n}\right] \in[0,1]^{n}$ such that

$$
\breve{x}_{i}(j)_{k}=\left\{\begin{array}{ll}
l_{i j} & k=j, \\
0 & \text { otherwise, }
\end{array} \quad \forall k \in J\right.
$$


The solution set of the $i$ th single fuzzy relation equation (4) could be characterized as below.

Theorem 1. Let $i \in$ I. If $S_{T_{D P}^{\gamma}}\left(a_{i}, b_{i}\right) \neq \emptyset$, then $S_{T_{D P}^{\gamma}}^{\gamma}\left(a_{i}, b_{i}\right)=\bigcup_{j \in J_{i}}\left[\breve{x}_{i}(j), \hat{x}_{i}\right]$.

According to Theorem $1, \hat{x}_{i}$ is the unique maximum solution of $S_{T_{D P}^{\gamma}}\left(a_{i}, b_{i}\right)$, while $\breve{x}_{i}(j)$ 's $\left(j \in J_{i}\right)$ are the minimal solutions.

Definition 3. Let $\hat{x}_{i}(i \in I)$ be the unique maximum solution of $S_{T_{D P}^{\gamma}}\left(a_{i}, b_{i}\right)$. We define $\bar{X}=\min _{i \in I} \hat{x}_{i}$.

Definition 4. Let $e: I \rightarrow J_{i}$ so that $e(i)=j \in J_{i}, \forall i \in I$. Let $E$ be the set of all vectors $e$. For the sake of convenience, we represent each $e \in E$ as an m-dimensional vector $e=\left[j_{1}, j_{2}, \cdots, j_{m}\right]$ in which $j_{k}=e(k)$.

Definition 5. Let $e=\left[j_{1}, j_{2}, \cdots, j_{m}\right] \in E$. We define $\underline{X}(e)=\left[\underline{X}(e)_{1}, \underline{X}(e)_{2}, \cdots, \underline{X}(e)_{n}\right] \in$ $[0,1]^{n}$, where $\underline{X}(e)_{j}=\max _{i \in I}\left\{\breve{x}_{i}(e(i))_{j}\right\}=\max _{i \in I}\left\{\breve{x}_{i}\left(j_{i}\right)_{j}\right\}, \forall j \in J$.

Theorem 2. $S_{T_{D P}^{\gamma}}(A, b)=\bigcup_{e \in E}[\underline{X}(e), \bar{X}]$.

Theorem 2 gives the complete characterization of the solution set of system (2). It follows from Theorem 2 that $\bar{X}$ is the unique maximum solution (if it is consistent), "and $\underline{X}(e)(e \in E)$ are the minimal solutions of $S_{T_{D P}^{\gamma}}(A, b)$ " [1]. Moreover, the consistency of system (2) could be checked by the feasibility of $\bar{X}$, according to the following Corollary 1 .

Corollary 1. (first necessary and sufficient condition) $S_{T_{D P}^{\gamma}}(A, b) \neq \emptyset$ if and only if $\bar{X} \in$ $S_{T_{D P}^{\gamma}}^{\gamma}(A, b)$.

\section{Main result}

In this section, we will show that some results presented in Ref. [1] are invalid for some specific cases regarding the system of max-Dubois-Prade fuzzy relation equations. In order to make sure the results valid for all cases, we point out the causing reason and further make some modifications of the definitions in [1].

\subsection{Phenomenon: $\underline{X}(e)$ might not be a (minimal) solution for some specific $e \in E$}

As mention above, it was pointed out in [1] that the vectors $\{\underline{X}(e): e \in E\}$ are the minimal solutions of system (2), i.e. $\underline{X}(e) \in S_{T_{D P}^{\gamma}}(A, b), \forall e \in E$. However it is found that this assertion is uncorrect in some specific case. In other words, there might exit some $e \in E$, such that $\underline{X}(e)$ is not a solution of (2).

Now we declare our assertions as follows:

(i) It is possible that $\underline{X}(e) \notin S_{T_{D P}^{\gamma}}(A, b)$ for some $e \in E$.

(ii) Even if $\underline{X}(e) \in S_{T_{D P}^{\gamma}}(A, b)$ holds for some $e \in E$, the solution $\underline{X}(e)$ might not be a minimal solution of system (2).

We take the example from [1] to support our above-mentioned assertions. 
Example 1. [1] Consider the problem below with Dubois-Prade t-norm

$$
\left[\begin{array}{cccccc}
0.9 & 0.4 & 0.6 & 0.7 & 0.4 & 0.4 \\
0.5 & 0.1 & 0.2 & 0.3 & 0.5 & 0.2 \\
0.2 & 0.8 & 0.4 & 0.4 & 0.6 & 0.9 \\
0.9 & 0.7 & 0.3 & 0.8 & 0.8 & 0.5 \\
0 & 0.4 & 0.2 & 0.2 & 0.1 & 0.7
\end{array}\right] \varphi x=\left[\begin{array}{l}
0.7 \\
0.5 \\
0.6 \\
0.8 \\
0.4
\end{array}\right]
$$

where $\varphi(x, y)=T_{D P}^{0.5}(x, y)=\frac{x y}{\max \{x, y, 0.5\}}$ (i.e. $\left.\gamma=0.5\right)$.

By simple computation, it could be obtained that $J_{1}=\{1,4\}, J_{2}=\{1,5\}, J_{3}=$ $\{2,5,6\}, J_{4}=\{1,4,5\}, J_{5}=\{2,6\}$. Maximum solutions of the equations in system (9) are

$$
\begin{aligned}
& \hat{x}_{1}=[0.7,1,1,1,1,1], \\
& \hat{x}_{2}=[1,1,1,1,1,1], \\
& \hat{x}_{3}=[1,0.6,1,1,1,0.6], \\
& \hat{x}_{4}=[0.8,1,1,1,1,1], \\
& \hat{x}_{5}=[1,1,1,1,1,0.4],
\end{aligned}
$$

respectively. On the other hand, all the minimal solutions are as

$$
\begin{aligned}
& \breve{x}_{1}(1)=[0.7,0,0,0,0,0], \breve{x}_{1}(4)=[0,0,0,0.7,0,0], \\
& \breve{x}_{2}(1)=[0.5,0,0,0,0,0], \breve{x}_{2}(5)=[0,0,0,0,0.5,0], \\
& \breve{x}_{3}(2)=[0,0.6,0,0,0,0], \breve{x}_{3}(5)=[0,0,0,0,0.6,0], \breve{x}_{3}(6)=[0,0,0,0,0,0.6], \\
& \breve{x}_{4}(1)=[0.8,0,0,0,0,0], \breve{x}_{4}(4)=[0,0,0,0.8,0,0], \breve{x}_{4}(5)=[0,0,0,0,0.8,0], \\
& \breve{x}_{5}(2)=[0,0.5,0,0,0,0], \breve{x}_{5}(6)=[0,0,0,0,0,0.4] .
\end{aligned}
$$

Based on $\hat{x}_{i}(i=1,2, \cdots, 5)$, it is easy to find the maximum solution of system (9), i.e.

$$
\bar{X}=\hat{x}_{1} \wedge \hat{x}_{2} \wedge \cdots \wedge \hat{x}_{5}=[0.7,0.6,1,1,1,0.4] .
$$

By Definition 4, $|E|=\left|J_{1}\right| \times\left|J_{2}\right| \times \cdots \times\left|J_{5}\right|=2 \times 2 \times 3 \times 3 \times 2=72$. As a consequence, the authors said "we have 72 solutions $\underline{X}(e)$ associated to 72 vectors $e$ " in [1].

In fact, this statement is incorrect. For example, take $e_{1}=[1,1,2,1,2] \in E$, then we have $\underline{X}\left(e_{1}\right)=\breve{x}_{1}(1) \vee \breve{x}_{2}(1) \vee \breve{x}_{3}(2) \vee \breve{x}_{4}(1) \vee \breve{x}_{5}(2)=[0.8,0.6,0,0,0,0]$. Substituting $\underline{X}\left(e_{1}\right)$ in system (9), it is found that the first equation doesn't hold. Hence $\underline{X}\left(e_{1}\right)$ is not a solution of (9).

Moreover, take $e_{2}=[4,1,5,5,2] \in E$. Even though the corresponding vector $\underline{X}\left(e_{2}\right)=$ $[0.5,0.5,0,0.7,0.8,0]$ is a solution of (9), it is not a minimal solution. It is easy to check that $x=[0,0.5,0,0.7,0.8,0]$ is also a solution of (9). But it holds that $x \leq \underline{X}\left(e_{2}\right)$ and $x \neq \underline{X}\left(e_{2}\right)$.

Next we will explain the reason causing the above conflict and provide some necessary modification to improve the results in [1]. 


\subsection{Reason: it is possible that $\underline{X}(e) \not \subset \bar{X}$}

What is the reason causing the non-membership relation $\underline{X}(e) \notin S_{T_{D P}^{\gamma}}(A, b)$ ?

In fact, whether $\underline{X}(e)$ belongs to $S_{T_{D P}^{\gamma}}(A, b)$ is determined by availability of the inequality that $\underline{X}(e) \leq \bar{X}$. Furthermore, we provide the following Theorem 3 to show the necessary and sufficient condition that ensures the vector $\underline{X}(e)$ to be a solution of (2), for a given $e \in E$.

Lemma 4. A vector $x \in[0,1]^{n}$ is a solution of system (2), if and only if for any $i \in I$, $a_{i j} \varphi x_{j}$ holds for all $j \in J$ and $a_{i j_{i}} \varphi x_{j_{i}}$ holds for some $j_{i} \in J$.

Proof. The proof is trivial.

Theorem 3. Let $e \in E$. Then $\underline{X}(e) \in S_{T_{D P}^{\gamma}}(A, b)$ if and only if $\underline{X}(e) \leq \bar{X}$.

Proof. Since $\bar{X}$ is the maximum solution, $\underline{X}(e) \in S_{T_{D P}^{\gamma}}(A, b) \Rightarrow \underline{X}(e) \leq \bar{X}$ is straightforward. Next we just verify the contrast implication. Assume that $\underline{X}(e) \leq \bar{X}$ for some $e \in E$. We have to prove that $\underline{X}(e) \in S_{T_{D P}^{\gamma}}(A, b)$.

Take arbitrary $i \in I$.

(i) By Lemma $4, \bar{X} \in S_{T_{D P}^{\gamma}}(A, b)$ implies that $a_{i j} \varphi \bar{X}_{j} \leq b_{i}, \forall j \in J$. On the other hand, the assumption that $\underline{X}(e) \leq \bar{X}$ indicates $\underline{X}(e)_{j} \leq \bar{X}_{j}, \forall j \in J$. Due to the monotonicity of the operator $\varphi$, we have

$$
a_{i j} \varphi \underline{X}(e)_{j} \leq a_{i j} \varphi \bar{X}_{j} \leq b_{i}, \forall j \in J
$$

(ii) Suppose $e=\left[j_{1}, j_{2}, \cdots, j_{m}\right]$. By Definition $5, \underline{X}(e)=\bigvee_{k \in I} \breve{x}_{k}\left(j_{k}\right)$. Thus

$$
\underline{X}(e)_{j_{i}}=\bigvee_{k \in I} \breve{x}_{k}\left(j_{k}\right)_{j_{i}} \geq \breve{x}_{i}\left(j_{i}\right)_{j_{i}}
$$

According to (8), it holds that

$$
\breve{x}_{i}\left(j_{i}\right)_{j_{i}}=l_{i j_{i}}
$$

Since $j_{i} \in J_{i}$, it follows from (7) that

$$
\bar{X}_{j_{i}}=\left(\bigwedge_{k \in I} \hat{x}_{k}\right)_{j_{i}}=\bigwedge_{k \in I}\left(\hat{x}_{k}\right)_{j_{i}} \leq\left(\hat{x}_{i}\right)_{j_{i}}=u_{i j_{i}}
$$

Combining (10), (11) and (12), we have

$$
l_{i j_{i}}=\breve{x}_{i}\left(j_{i}\right)_{j_{i}} \leq \underline{X}(e)_{j_{i}} \leq \bar{X}_{j_{i}} \leq u_{i j_{i}} .
$$

According to Lemma 2, it holds that $a_{i j_{i}} \varphi \underline{X}(e)_{j_{i}}=b_{i}$.

By Lemma 4, the above-proved (i) and (ii) contribute to $\underline{X}(e) \in S_{T_{D P}^{\gamma}}(A, b)$. 


\subsection{Correction: modification of the index set $J_{i}$}

In this subsection we aim to make some modification for improving the results in [1]. To make the above-mention assertion presented in [1] correct, we just need to modified the index set $J_{i}$ used in Definition 4.

For arbitrary $i \in I$, let

$$
J_{i}^{=}=\left\{j \in J_{i} \mid l_{i j} \leq \bar{X}_{j}\right\}
$$

It is clear that $J_{i}^{=} \subseteq J_{i}, \forall i \in I$. Moreover, it is possible that $J_{i}^{=} \neq J_{i}$, e.g. $J_{3}^{=}$and $J_{4}^{=}$are not equal to $J_{3}$ and $J_{4}$, respectively, in Example 2 .

We replace $J_{i}$ in Definition 4 by $J_{i}^{=}$in (14). Then the Definition 4 turns out to be Definition 4' as below.

Definition 4'. Let $e: I \rightarrow J_{i}^{=}$so that $e(i)=j \in J_{i}^{=}, \forall i \in I$. Let $E^{=}$be the set of all vectors $e$. For the sake of convenience, we represent each $e \in E^{=}$as an m-dimensional vector $e=\left[j_{1}, j_{2}, \cdots, j_{m}\right]$ in which $j_{k}=e(k)$.

The set $E^{=}$could be simply viewed as

$$
E^{=}=J_{1}^{=} \times J_{2}^{=} \times \cdots \times J_{m}^{=}
$$

Based on the modified Definition 4', we further provide a theorem to illustrate the correction.

Theorem 4. Let $e=\left[j_{1}, j_{2}, \cdots, j_{m}\right] \in E^{=}$, where $E^{=}$is as defined in Definition 4'. Then the corresponding vector

$$
\underline{X}(e)=\left[\underline{X}(e)_{1}, \underline{X}(e)_{2}, \cdots, \underline{X}(e)_{n}\right]=\left[\bigvee_{i \in I} \breve{x}_{i}\left(j_{i}\right)_{1}, \bigvee_{i \in I} \breve{x}_{i}\left(j_{i}\right)_{2}, \cdots, \bigvee_{i \in I} \breve{x}_{i}\left(j_{i}\right)_{n}\right]=\bigvee_{i \in I} \breve{x}_{i}\left(j_{i}\right)
$$

is a solution of system (2), i.e. $\underline{X}(e) \in S_{T_{D P}^{\gamma}}(A, b)$.

Proof. According to Definition 5, $\underline{X}(e)=\breve{x}_{1}\left(j_{1}\right) \vee \breve{x}_{2}\left(j_{2}\right) \vee \cdots \vee \breve{x}_{m}\left(j_{m}\right)$. By (8), for arbitrary $i \in I$, there exits at most one nonzero component in the vector $\breve{x}_{i}\left(j_{i}\right)$. If there exists, the unique non-zero component is indeed the $j_{i}$ th component, i.e. $\breve{x}_{i}\left(j_{i}\right)_{j_{i}}=l_{i j_{i}} \neq 0$. Notice that $\bar{X}_{j} \geq 0$ for all $j \in J$. Moreover, since $j_{i} \in J_{i}^{=}$, it follows from (14) that $\breve{x}_{i}\left(j_{i}\right)_{j_{i}}=l_{i j_{i}} \leq \bar{X}_{j_{i}}$. As a result, we have $\breve{x}_{i}\left(j_{i}\right) \leq \bar{X}$. Due to the arbitrariness of $i$, we further get $\underline{X}(e)=\breve{x}_{1}\left(j_{1}\right) \vee \breve{x}_{2}\left(j_{2}\right) \vee \cdots \vee \breve{x}_{m}\left(j_{m}\right) \leq \bar{X}$. According to Theorem $3, \underline{X}(e)$ is a solution of system (2)

Example 2. Continue to discuss the system (9) in Example 1, based on the modified Definition 4'.

According to (14), we get

$$
J_{1}^{=}=\{1,4\}, J_{2}^{=}=\{1,5\}, J_{3}^{=}=\{2,5\}, J_{4}^{=}=\{4,5\}, J_{1}^{=}=\{2,6\} .
$$

Hence, the cardinality of $E^{=}$is 


$$
\left|E^{=}\right|=J_{1}^{=} \times J_{2}^{=} \times \cdots \times J_{5}^{=}=2^{5}=32 .
$$

That is to say, there are 32 solutions corresponding the 32 vectors $e$ in $E^{=}$. These 32 solutions could be represented by

$$
\left\{\underline{X}(e) \mid e \in E^{=}\right\} \text {. }
$$

Recall that in Ref. [1], one should compute 72 vectors based on $E$. But by our modified set $E^{=}$, we just need to compute 32 ones. It is much easier to compute the solution set of system (9).

\section{Conclusion}

Max-Dubois-Prade fuzzy relation equations system with parameter $\gamma$ and its corresponding optimization problem were recently investigated in [1]. For characterizing the solution set of system (2), the authors constructed the index set $E=J_{1} \times J_{2} \times \cdots \times J_{m}$ and asserted that all vectors in $\{\underline{X}(e): e \in E\}$ are minimal solutions. However, it is found that $\underline{X}(e)$ might not be a solution for some specific $e \in E$. In order to make every vector $\underline{X}(e)$ be a solution of the corresponding fuzzy relation system, we modify the index set $E$ to $E^{=}=J_{1}^{=} \times J_{2}^{=} \times \cdots \times J_{m}^{=}$. Moreover, we have verified that for each $e \in E^{=}$, the corresponding vector $\underline{X}(e)$ is a solution of system (2). Finding the solution set based on $E^{=}$should be easier than that based on $E$.

The limitation of this study and Ref. [1] is that the proposed resolution algorithm is only able to find an approximate (optimal) solution of the relevant optimization problem, but not an exact solution. Moreover, Ref. [1] hasn't explained the influence of the parameter $\gamma$ to the resolution process or results. In our future works, there are some interesting researching topics, including but not limited to (i) the influence of the parameter $\gamma$, (ii) the sensitivity analysis with respect to $A$ or/and $b$; (iii) the optimization problems subject to other kinds of fuzzy relation systems; (iv) the potential industrial applications of the fuzzy relation optimization problems.

\section{References}

[1] A. Ghodousian, M. Naeeimi, A. Babalhavaeji, Nonlinear optimization problem subjected to fuzzy relational equations defined by Dubois-Prade family of t-norms, Computers \& Industrial Engineering 119 (2018) 167-180.

[2] E. Bartl, Minimal solutions of generalized fuzzy relational equations: Probabilistic algorithm based on greedy approach, Fuzzy Sets and Systems 260 (2015) 25-42.

[3] A. A. Molai, Resolution of a system of the max-product fuzzy relation equations using LoUfactorization, Information Sciences 234 (2013) 86-96.

[4] X.-P. Yang, H.-T. Lin, X.-G. Zhou, B.-Y. Cao, Addition-min fuzzy relation inequalities with application in BitTorrent-like Peer-to-Peer file sharing system, Fuzzy Sets and Systems 343 (2018) 126-140.

[5] X.-B. Yang, X.-P. Yang, K. Hayat, A New Characterisation of the Minimal Solution Set to Max-min Fuzzy Relation Inequalities, Fuzzy Information and Engineering 9 (2017) 423-435.

[6] S.-J. Yang, Some Results of the Fuzzy Relation Inequalities With Addition-Min Composition, IEEE Transactions on Fuzzy System 26(1) (2018) 239-245.

[7] A. Ghodousian, A. Babalhavaeji, An efficient genetic algorithm for solving nonlinear optimization problems defined with fuzzy relational equations and max-Łukasiewicz composition, Applied Soft Computing 69 (2018) 475-492. 
[8] S.-C. Fang, G. Li, Solving fuzzy relation equations with a linear objective function, Fuzzy Sets and Systems 103 (1999) 107-113.

[9] A. Ghodousian, E. Khorram, Solving a linear programming problem with the convex combination of the max-min and the max-average fuzzy relation equations, Applied Mathematics and Computation 180 (2006) 411-418.

[10] S.-M. Guu, Y.-K. Wu, A Linear Programming Approach for Minimizing a Linear Function Subject to Fuzzy Relational Inequalities With Addition-Min Composition, IEEE Transactions on Fuzzy System 25(4) (2017) 985-992.

[11] S.-J. Yang, An algorithm for minimizing a linear objective function subject to the fuzzy relation inequalities with addition-min composition, Fuzzy Sets and Systems 255 (2014) 41-51.

[12] X.-P. Yang, Optimal-vector-based algorithm for solving min-max programming subject to addition-min fuzzy relation inequality, IEEE Transactions on Fuzzy Systems 25(5) (2017) 1127-1140.

[13] J. Lu, S.-C. Fang, Solving nonlinear optimization problems with fuzzy relation equations constraints, Fuzzy Sets and Systems 119 (2001) 1-20.

[14] E. Khorram, R. Ezzati, Z. Valizadeh, Solving nonlinear multi-objective optimization problems with fuzzy relation inequality constraints regarding Archimedean triangular norm compositions, Fuzzy Optimization and Decision Making 11 (2012) 299-335.

[15] X.-P. Yang, X.-G. Zhou, B.-Y. Cao, Single-variable term semi-latticized fuzzy relation geometric programming with max-product operator, Information Sciences 325 (2015) 271-287.

[16] X.-P. Yang, Linear programming method for solving semi-latticized fuzzy relation geometric programming with max-min composition, International Journal of Uncertainty, Fuzziness and Knowledge-Based Systems 23 (2015) 781-804.

[17] E. Shivanian, E. Khorram, Monomial geometric programming with fuzzy relation inequality constraints with max-product composition, Computer \& Industrial Engineering 56 (2009) 1386-1392.

[18] Y.-K. Wu, Optimizing the geometric programming problem with single-term exponents subject to maxmin fuzzy relational equation constraints, Mathematical and Computer Modelling 47 (2008) 352-362.

[19] X.G. Zhou, R. Ahat, Geometric programming problem with single-term exponents subject to maxproduct fuzzy relational equations, Mathematical and Computer Modelling 53 (2011) 55-62.

[20] A. Abbasi Molai, A new algorithm for resolution of the quadratic programming problem with fuzzy relation inequality constraints, Computers \& Industrial Engineering 72 (2014), 306-314.

[21] J. Loetamonphong, S.-C. Fang, Optimization of fuzzy relation equations with max-product composition, Fuzzy Sets and Systems 118 (2001) 509-517.

[22] B. Hedayatfar, A. Abbasi Molai, S. Aliannezhadi, Separable programming problems with the maxproduct fuzzy relation equation constraints, Iranian Journal of Fuzzy Systems, 16(1) (2019) 1-15.

[23] S. Aliannezhadi, A. Abbasi Molai, Geometric programming with a single-term exponent subject to bipolar max-product fuzzy relation equation constraints, Fuzzy Sets and Systems 397 (2020) 61-83.

[24] X.-P. Yang, D.-H. Yuan, B.-Y. Cao, Lexicographic optimal solution of the multi-objective programming problem subject to max-product fuzzy relation inequalities, Fuzzy Sets and Systems 341 (2018) 92-112.

[25] X.-P. Yang, X.-G. Zhou, B.-Y. Cao, Latticized linear programming subject to max-product fuzzy relation inequalities with application in wireless communication, Information Sciences 358C359 (2016) 44-55.

[26] J. Qiu, H. Xue, G. Li, X. Yang, Fuzzy relation bilevel optimization model in the wireless communication station system, IEEE Access, 8 (2020) 60811-60823.

[27] F.-F. Guo, J. Shen, A Novel Smoothing Approach for Linear Objective Optimizations Subject to Fuzzy Relation Inequalities With Addition-Min Composition, IEEE Transactions on Fuzzy System 29(8) 2021 2444-2450.

[28] Y.-L. Chiu, S.-M. Guu, J. Yu, Y.-K. Wu, A single-variable method for solving min-max programming problem with addition-min fuzzy relational inequalities, Fuzzy Optimization and Decision Making 18 (2019) 433-449.

[29] X. Yang, J. Qiu, H. Guo, X. Yang, Fuzzy relation weighted minimax programming with addition-min composition, Computers \& Industrial Engineering 147 (2020) 106644.

[30] S.-M. Guu, Y.-K. Wu, Multiple objective optimization for systems with addition-min fuzzy relational inequalities, Fuzzy Optimization and Decision Making 18 (2019) 529-544.

[31] X.-P. Yang, Z.-F. Hao, Supereigenvalue problem to addition-min fuzzy matrix with application in P2P file sharing system, IEEE Transactions on Fuzzy System 28(8) (2020) 1640-1651.

[32] X.-P. Yang, Leximax minimum solution of addition-min fuzzy relation inequalities, Information Sciences 524 (2020) 184-198. 